\title{
ldentification du gène de la granulomatose chronique
}

Les nouvelles
de ce numéro
ont été préparées par :

A. Kahn

J.-C. Dreyfus

J.-C. Kaplan

J.-P. Grünfeld
I. Royer-Pokora B, Kunkel LM, Monaco AP, et al. Cloning the gene for an inherited human disorder, chronic granulomatous discase on the basis of its chromosomal location. Nature 1986; $322: 32-8$.
La granulomatose chronique léthale de l'enfant est une maladie héréditaire dont la forme la plus fréquente est transmise selon le mode récessif lié au sexe : les garçons sont atteints, les femmes étant transmettrices. Les symptômes comportent une sensibilité aux infections microbiennes et fongiques conduisant immanquablement à la mort. Les phagocytes du jeune malade englobent normalement les germes mais ne les détruisent pas, probablement parce qu'ils sont incapables de stimuler, lors de la phagocytose, la production d'anion superoxyde et de peroxydes. La base biochimique de cette altération fonctionnelle des leucocytes n'est pas connue, la seule anomalie identifiée étant l'absence d'une protéine héminique de spectre similaire à celui des cytochromes b. L'existence d'observations comportant des délétions entrainant à la fois une granulomatose chronique et une myopathie avait permis de déterminer que les gènes de ces deux maladies étaient liés et situés dans la bande p2I du chromosome X. Les efforts considérables consentis pour identifier le gène de la myopathie de Duchenne (DMD en anglais, pour Duchenne Muscular Dystrophy) eurent dès lors de rapides retombées sur l'étude du gène de la granulomatose (CGD en anglais, pour Chronic Granulomatous Disease) qui fut isolé par une approche uniquement génétique selon la stratégie générale suivante [I] :

Étape I : Des sondes génétiquement liées au locus " DMD " hybrident avec l'ADN normal mais pas avec celui d'un malade atteint de "CGD"; elles sont utilisées pour cribler une banque d'ADN génomique normal.

Étape 2 : Plusieurs clones sont ainsi détectés; leur ADN est immobilisé sur des filtres qui sont hybridés avec la copie ADN des ARNm de cellules supposées contenir le produit du gène CGD (lignée granulo-monocytaire). Deux clones sont positifs.

Étape 3: L'ADN génomique identifié à l'étape 2 est utilisé pour cribler une banque d'ADNc de cellules granulo-monocytaire. Des copies ADN du messager sont isolées et leur séquence nucléotidique est déterminée.

Étape 4: On vérifie que l'ADNc isolé à l'étape précédente hybride avec un messager de $5 \mathrm{~kb}$ présent dans les cellules phagocytaires normales mais absent dans les cellules de la plupart des observations de "CGD ». De plus, chez un malade atteint de "CGD ", cette sonde d'ADNc détecte une délétion génomique de quelques centaines de bases.

La séquence en acides aminés déduite de la séquence nucléotidique de l'ADNc correspond à une protéine de 54000 de poids moléculaire qui pourrait être transmembranaire et glycosylée et qui ne ressemble à aucune autre connue actuellement, notamment pas aux cytochromes b. Il est maintenant certain que l'obtention par génie génétique d'une grande quantité de la protéine dont le déficit est responsable de la granulomatose permettra d'en déterminer l'activité biologique, probablement enzymatique, ce qui devrait définitivement établir les bases biochimiques de la maladie. D'un autre point de vue cette découverte marque aussi une date dans l'histoire de la génétique et de la pathologie moléculaires : c'est la première fois qu'un gène responsable d'une maladie humaine est identifié, non grâce aux connaissances acquises sur son produit, mais à partir des informations recueillies sur sa localisation chromosomique et sa transmission génétique. Un tel isolement du gène impliqué dans la maladie permet secondairement d'analyser la structure et la fonction de la protéine pour laquelle il code... et par conséquent de comprendre les mécanismes de l'affection. Nul doute que d'autres exemples remarquables ne tarderont pas à illustrer cette nouvelle démarche, concernant la myopathie de Duchenne, la fibrose kystique du pancréas et la chorée de Hungtington notamment.

A. $K$. 